\title{
Automatic Synchronization Unit For The Parallel Operation Of Synchronous GENERATORS
}

\author{
Erdal Bekiroglu ${ }^{1}$, Member, IEEE \\ Alper Bayrak ${ }^{2}$, Student Member, IEEE \\ ${ }^{1}$ Abant Izzet Baysal University, Faculty of Engineering and Architecture, Bolu, Turkey bekiroglu_e@ibu.edu.tr \\ ${ }^{2}$ Izmir Institute of Technology, Faculty of Engineering, Izmir, Turkey alperbayrak@iyte.edu.tr
}

\begin{abstract}
In this study, an automatic synchronization unit has been developed for the parallel connection of synchronous generators. Two synchronous generators are connected in parallel automatically with the developed control unit. Synchronous generators are also connected in parallel with the line. The voltages, frequencies, phase sequences and synchronism time data have been transferred to the microcontroller. These data are monitored and evaluated by the control algorithm coded into the microcontroller. Parallel operation of generators are realized automatically when all parallel connection conditions are occur. The system doesn't require additional measuring tools for monitoring and control processes. The developed automatic synchronization unit is fast, cost effective, reliable and precise to be used for monitoring, measurement and parallel operation of the synchronous generators.
\end{abstract}

Index Terms: Synchronization, synchronous generators, parallel connection, microcontroller.

\section{INTRODUCTION}

Electrical power systems consist of the interconnection of large numbers of synchronous generators operating in parallel, interconnected by transmission lines, and supplying large numbers of widely distributed loads. When a synchronous generator ( $\mathrm{SG}$ ) is connected to an interconnected system containing many other SGs, the voltage and frequency at its terminals are fixed by the system [1].

Some of the benefits of operating multiple generators in parallel include increased reliability, expandability, flexibility, serviceability and efficiency. The redundancy inherent in parallel power generation provides significantly greater reliability for critical loads. In a parallel configuration, if one generator fails, the most critical loads are redistributed among the other units in the system. Utilizing multiple smaller generators instead of a single large unit offers greater location flexibility. With multiple generators available, individual units can be taken out of service for repair or maintenance without losing standby power for critical circuits. Electrical machines operate at maximum efficiency value close to the rated load. If the load is too low, the efficiency of the SG will decrease. Parallel operation allows operating SGs around their rated load resulting operating with high efficiency $[2,3]$.

Variety studies related to parallel operation and control of SGs have presented in the literature. These studies include simulation, power control, synchronization and stability of power system including SGs [4, 5]. A standard of synchronization used for power systems is given in [6]. Simulation of parallel connected SGs is presented in [7]. An automatic digital synchronization system has been proposed in [8]. Using sensors and PLC in the control unit increase the cost of the system. A control unit for the parallel operation of AC Generators is developed and presented in [9]. Zero crossing detectors are used for transmit voltage signals of generators to the microcontroller.

In this study, microcontroller based an automatic synchronization unit has been developed for the parallel operation of SGs. The control unit read calculates and evaluates the frequency, voltage, phase sequence of the received input signals and then provides the synchronization for the monitoring parallel connection conditions and parallel operation of generators. The program coded into the microcontroller has developed effectively to eliminate the interface electronics circuit from the system.

Parallel operation of generators is achieved automatically with the control unit when all parallel connection conditions occur. There is no need to use measuring tools, such as voltmeters, frequency meters, phase sequence meter, and synchroscope for monitoring the parallel connection conditions. The proposed automatic synchronization unit is cost effective, quick, reliable, stable and accurate to be used for monitoring, measurement and parallel operation of the SGs.

\section{PARALLEL OPERATION OF THE SGs}

SGs are connected in parallel to feed bigger loads and improve reliability of the electrical power systems. When required conditions are occur a SG can be connected in parallel with another SG or with transmission line. The parallel conditions required for the parallel connection of SGs are given as follows:

1. Frequencies should be equal $(f l=f 2)$

2. Voltages should be equal $(V l=V 2)$

3. Phase sequence of the SGs should be same (Direction of the rotating field should be same)

4. Parallel connection should be realized at the synchronism time. 
In the classical systems; frequencies are measured with frequency meters, voltages are measured with voltmeters, phase sequence is measured with phase sequence meter, and synchronism time is measured with synchroscope. In the presented study, all of the measurement tools are eliminated from the system. The developed control unit measures required signals of the SGs and determines parallel connection time when all conditions are provided. The hardware implementation of the parallel operation system with classical measurement tools is given in Fig. 1.

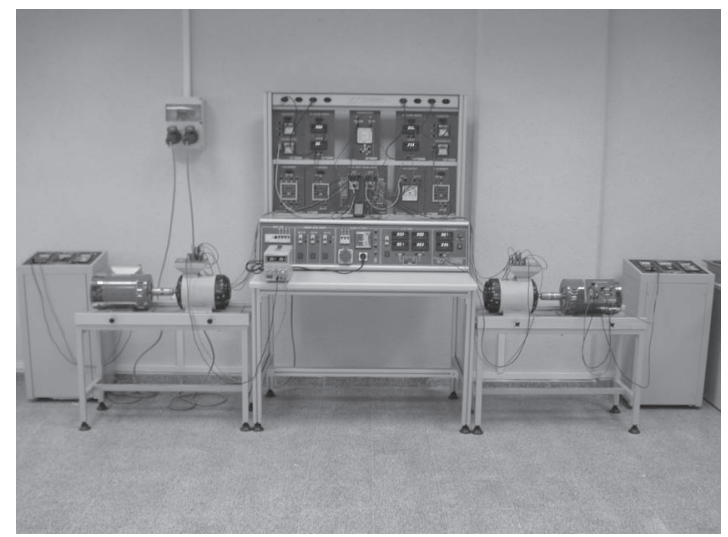

Fig.1 - Hardware implementation of the parallel operation system of SGs

\section{AUTOMATIC SYNCHRONIZATION UNIT}

To eliminate measurement tools and to obtain simplified, precise and quick parallel connection for SGs a PIC microcontroller based automatic synchronization monitoring and control unit has been designed. The main block diagram of the microcontroller based parallel connection system is given in Fig. 2. As seen from the figure, voltage signals of the SGs are transmitted to the microcontroller.

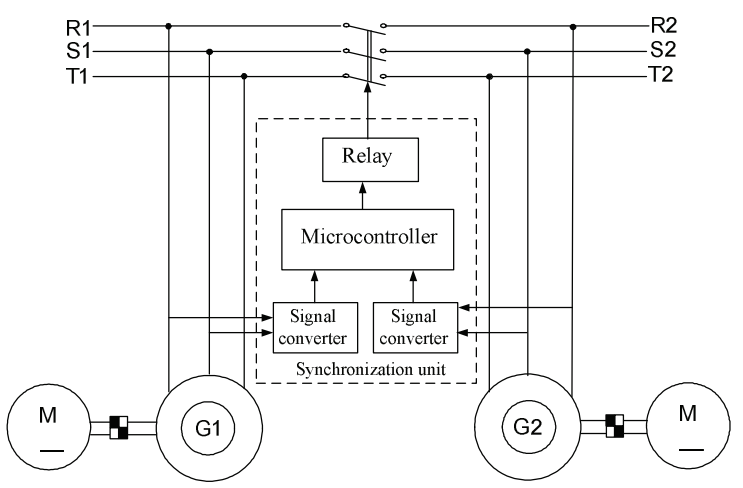

Fig. 2 - Block diagram of the microcontroller based parallel connection system
Transformers are used to convert phase voltages to the lower voltages that can be processed with microcontroller. Microcontroller converts these signals and then calculates the frequencies, voltages, phase sequence of the SGs. If these three conditions are provided, microcontroller captures synchronism time between phase voltages of the SGs. When synchronization occurs the microcontroller sends the control signal to the relay and parallel connection switch is turned on for parallel operation. The designed automatic synchronization control unit has been shown in Fig. 3.

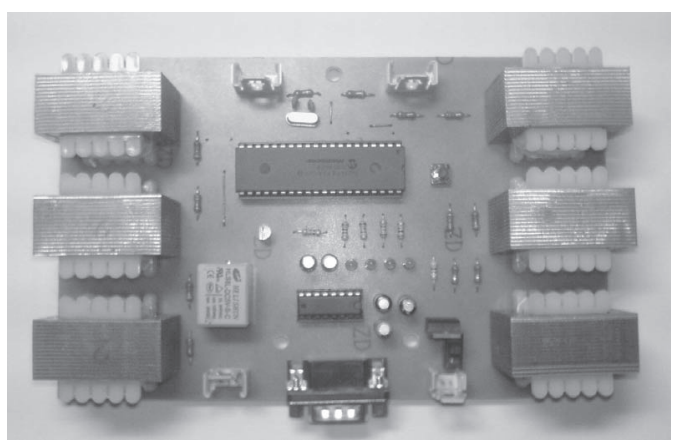

Fig. 3 - A photograph of the automatic synchronization unit

A single 16F877 PIC microcontroller has been used in the control system. Microcontroller has been operated with $4 \mathrm{MHz}$ oscillator. Loop time of each command is $1 \mu \mathrm{s}$. Comparator, ADC, Timer- 1 modules and $\mathrm{A}, \mathrm{B}, \mathrm{E}$ ports of the microcontroller have been used in the application. Port configuration of the microcontroller used for the study is shown $n$ Fig. 4.

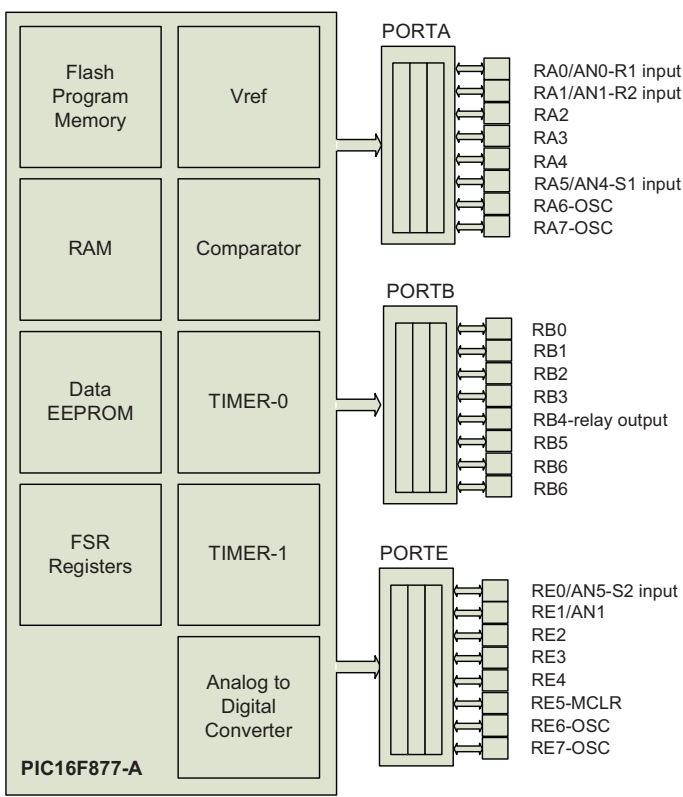

Fig. 4 - Port configuration of the microcontroller 
Ports $\mathrm{A}$ and $\mathrm{E}$ have been set as analogous inputs and port-B is set as digital outputs. R1 and R2 phases of the two synchronous generators are connected to the number 0 and 1 inputs of the port- $\mathrm{A}$, and S1 and $\mathrm{S} 2$ phases are connected to the number 5 input of port$\mathrm{A}$ and number 0 input of port-E, respectively. The control unit doesn't require the T-phases of the generators. A relay realizing the parallel connection is connected to the number 4 input of the port B. Control unit read, calculate and evaluate the frequency, voltage, phase sequence of the received input signals and then provide the synchronization for the parallel operation of generators.

The flowchart of the main software program coded into the microcontroller for the parellel operation of the SGs is shown in Fig. 5. As shown from the flowchart; frequency, voltage, and phase sequence of the each SG is determined, respectively and when all the conditions occur, the synchronism time is searched to achieve parallel operation of the system.

Each parallel connection condition has been determined by sub-routine of the software programme. Detailed informations about sub-routines are given as follows, individullly.

Frequency calculation; The signals received from the $\mathrm{R}$ phases of the generators are checked for the calculation of frequencies. Comparator module of the microcontroller is used in this stage. Input signal is compared with internal produced reference voltage $V_{r e f}=0$ and then converted to the square wave signal. This square wave signal takes logic 1 and 0 data Microcontroller run the Timer-1 when the value of signal is 1 and stop the Timer- 1 when the signal rising from the 0 to the 1 once more. This is a period of the signal. Microcontroller read value of the counter and calculates the frequency from the loop time. Flowchart of the frequency calculation is given in Fig. 6 .

Voltage calculation; The signals received from the $\mathrm{R}$ phases of the generators are used for the calculation of voltages. The received signals are converted to the square wave signals as described in the calculation of the frequency. Microcontroller captures point of the square wave falling from the 1 to the 0 . The reference signal is bigger than the reference signal in the period of square wave has a 0 value. In the other words, the input signal is in the positive period. Microcontroller receives approximately 300 samples from the signal until the square wave rising to the 1 once again. Each received sample compared with the previous sample and the bigger value is saved as a new value. As a result, the peak value of the half period of the input signal is obtained. Then the rms value of the voltage is calculated. Flowchart of the voltage calculation is given in Fig. 7.

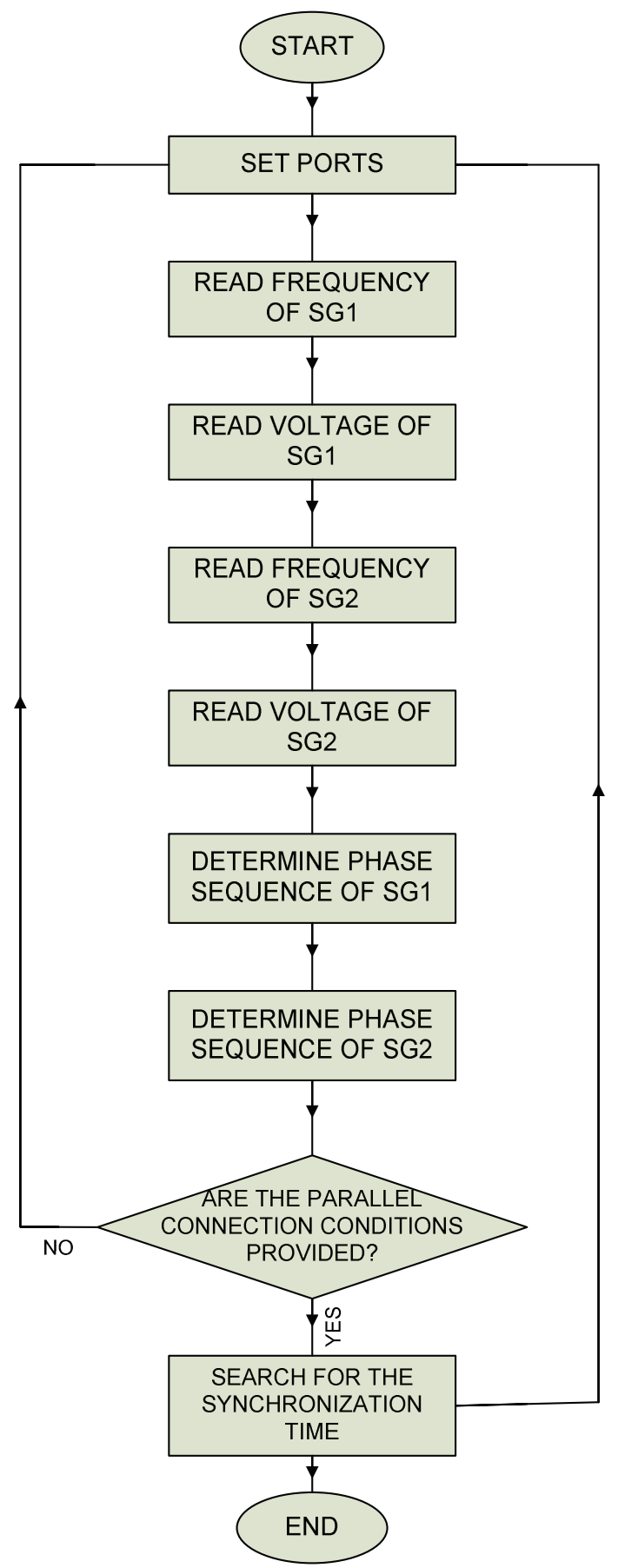

Fig. 5 - Flowchart of the main software program 


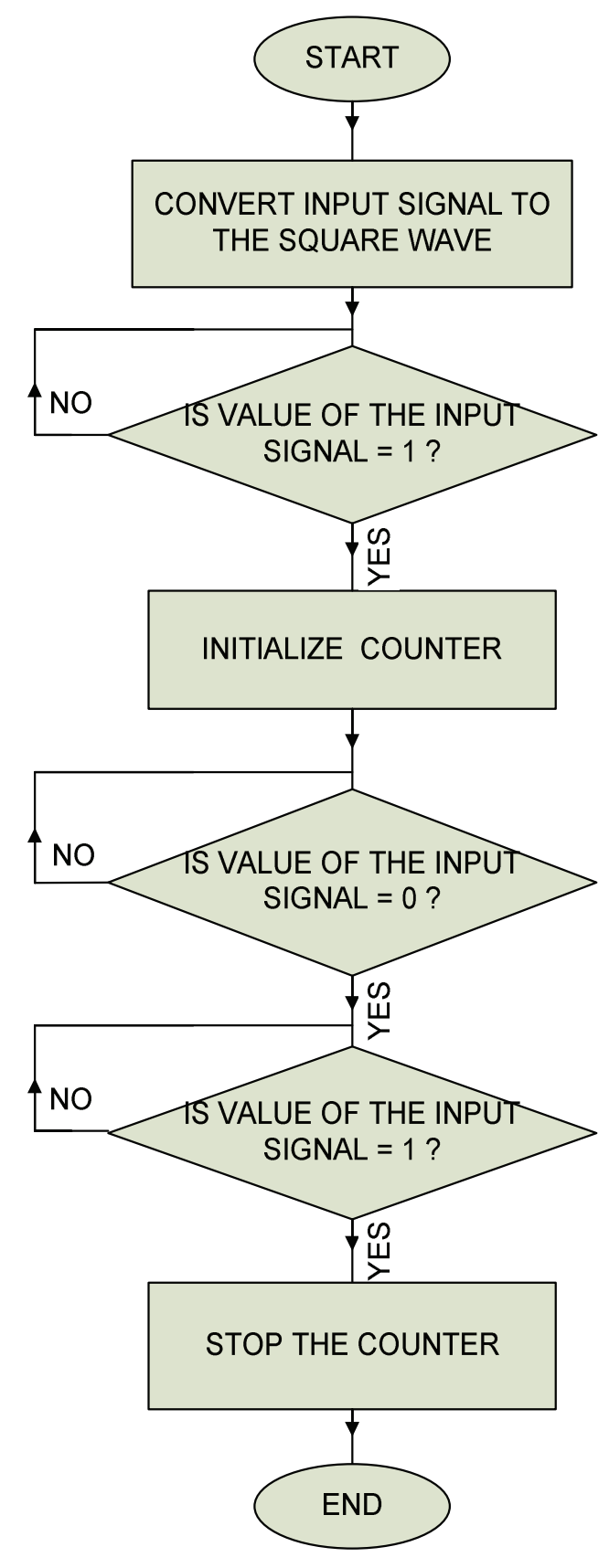

Fig. 6 - Flowchart of the frequency calculation loop

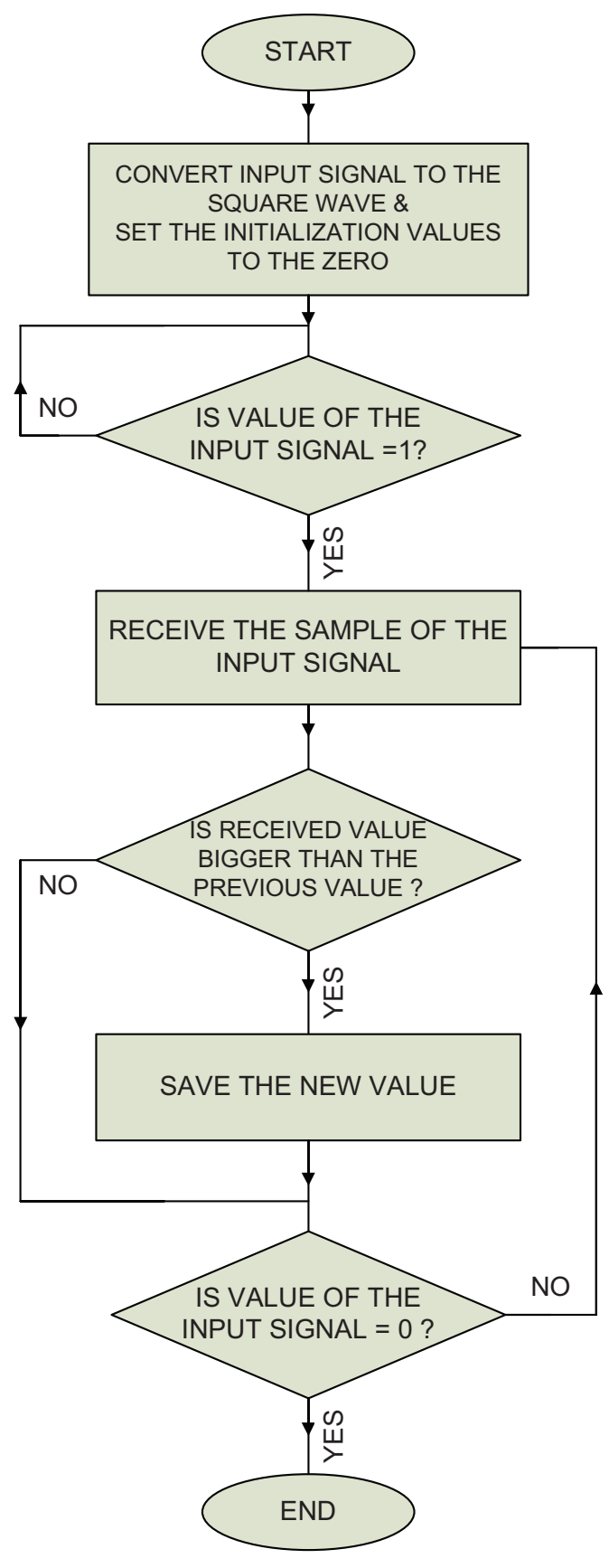

Fig. 7 - Flowchart of the voltage calculation loop

Determination of the phase sequence; The signals received from the $\mathrm{R}$ and $\mathrm{S}$ phases of the generators are used for the determination of the phase-sequence. The received signals are converted to the square wave signals. Microcontroller captures point of the square wave falling from the 1 to the 0 and wait $90^{\circ}+120^{\circ}$ beginning from that point. Controller captures the peak value of the phase- $\mathrm{R}$ with the $90^{\circ}$ waiting period and then checks the peak value of the phase-S has peak value or not at the $120^{\circ}$ forward. According to 
the calculation, the controller determining the phase sequence direction as 0 or 1 . If the phase sequence is determined as 0 , the phase sequence is in $\mathrm{CW}$ direction, else the phase sequence is determined as 1 and the phase sequence is in $\mathrm{CCW}$ direction Flowchart of the phase sequence determination is given in Fig. 8.

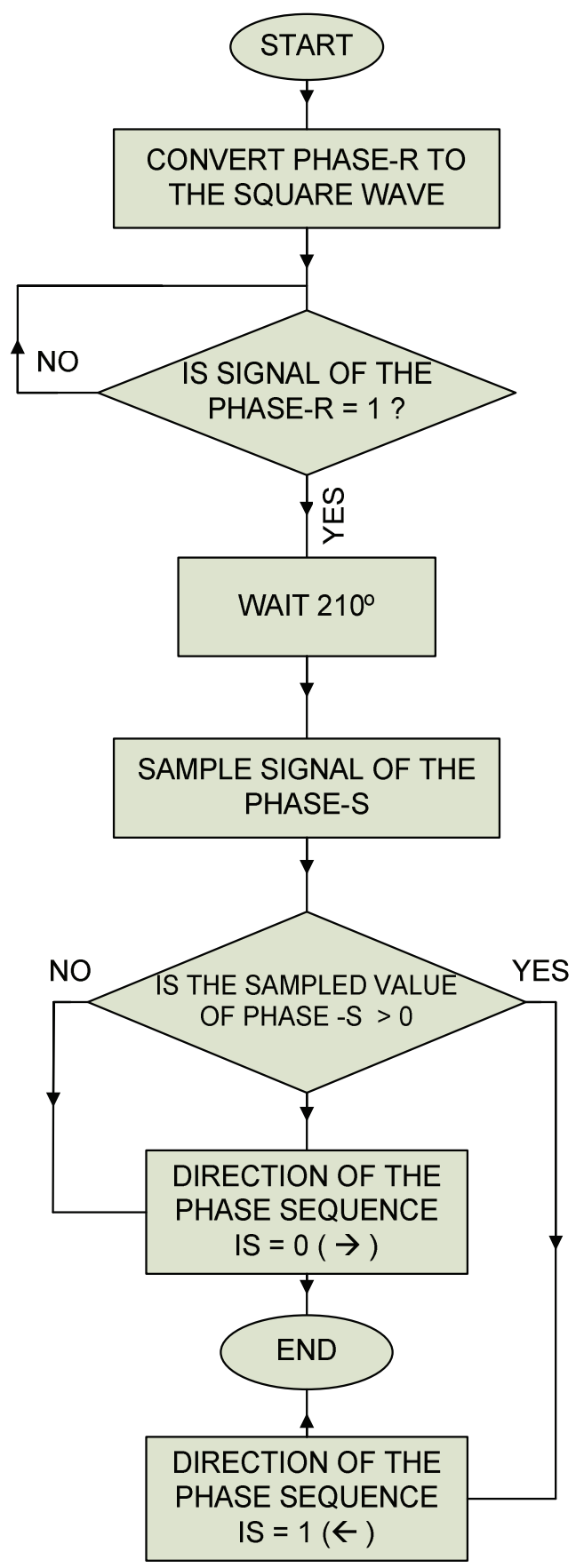

Fig. 8 - Flowchart of the phase sequence determination loop
Synchronism time; Synchronization algorithm is operated when all of the parallel connection conditions are provided. The signals received from the $\mathrm{R}$ phases of the generators are used for the detection of the synchronization. Both R-phases of the generators are converted to the square wave signals. Microcontroller explores the rising point from 0 to the 1 value simultaneously for two signals. The synchronism point is captured when the two phase are rising to 1 at the same time and the microcontroller sends control signal to the number 4 input of the port B to achieve parallel operation of generators with the relay. Flowchart of the synchronism time calculation is given in Fig. 9.

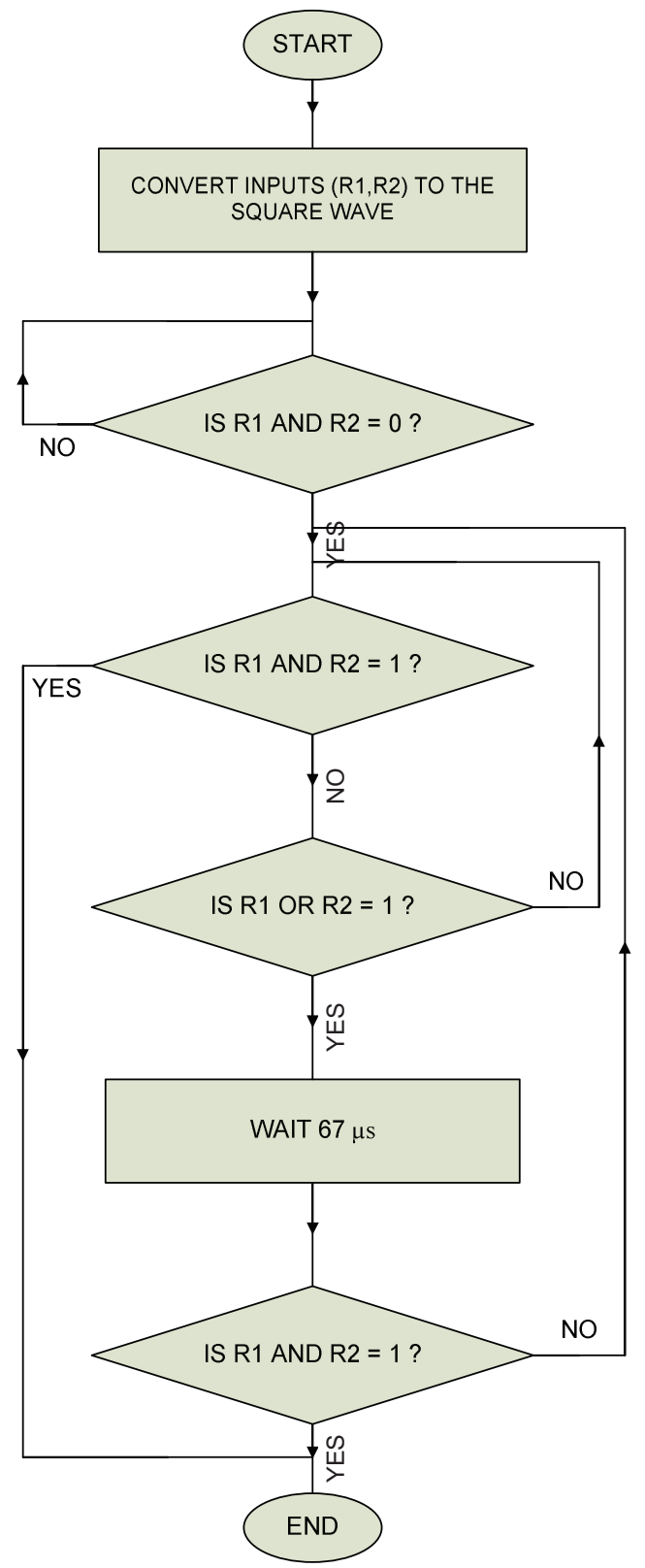

Fig. 9 - Flowchart of the synchronism time calculation loop 


\section{IV.CONCLUSIONS}

PIC16F877 microcontroller based an automatic synchronization unit has been achieved for the monitoring parallel connection conditions and parallel operation of SGs. Phase voltages of the SGs are transmitted to the microcontroller by the 220/5Vsignal transformers. There is no need for any interface electronics circuit for processing, converting and then transmitting signals to the microcontroller. All signals are transmitted to the microcontroller directly. Microcontroller calculates phase voltages, frequencies and phase sequences of the SGs according to the received signals. When all of the parallel connection conditions are exist, the microcontroller search for synchronism time to produce signal for the parallel operation. The developed system is simple and reliable. It is independent from extra measurement tools. As a result it is cost effective and can be used easily for the SGs parallel operation. It can also be used for the educational purposes.

\section{ACKNOWLEDGMENTS}

This work was supported from the Scientific Research Project Commision of the Abant Izzet Baysal University, under grant 2006.01.09.252.

\section{REFERENCES}

[1]. Fitzgerald, A.E, Kingsley, C., Umans, S.D., Electric Machinery, McGraw-Hill Book Company, Singapore, 1992.

[2]. Colak I., Synchronous Machines, Seckin Press, Ankara, 2003, In Turkish

[3]. Generac Power Systems, Technical Perspective, Generator Paralleling, 1-4.

[4]. Mahmoud M. S., Ismail A., Control of electric power systems, Systems Analysis Modeling Simulation, 43(12) (2003). p. 1639-1673.

[5]. Metwally H.M.B. Operation of new variable speed constant voltage and frequency generator connected to the grid, Energy Conversion and Management, 41(7), (2000) p. 701-712.

[6]. IEEE Std C37.118-2005, IEEE Power Engineering Society, IEEE standard for synchrophasors for power systems, 2005.

[7]. Çolak I., Yılmaz E.N., Simulation of the parallel connection of electrical power stations, IJEEE International Journal of Electrical Engineering Education, 36 (4) (1999). p. 332-341,

[8]. Emam, S.E.A., Automatic digital synchronization, International Conference on Electrical, Electronic and Computer Engineering, ICEEC'04, (2004). p. 778-784.

[9]. Çolak, İ., Bayındır, R., Sefa, İ., Demirbaș, S., Bal, G., Developing of a control unit for the parallel operation of AC generators, TPE'06 3rd International Conference on Technical and Physical Problems in Power Engineering, (2006). p. 134-137.
Erdal Bekiroglu received his BSc, $\mathrm{MSc}$ and $\mathrm{PhD}$ degrees in the Electrical Technologies Education from the Gazi University. He worked as a research assistant at Gazi University between 1996 and 2003. He is currently assistant professor at Department of Electrical and Electronics Engineering, Faculty of Engineering and Architecture, Abant Izzet Baysal University. His research interests are fuzzy logic, digital signal processors, drive and control of electrical machines.

Alper Bayrak received his BSc and MSc degrees in Electrical and Electronics Engineering Department from Karadeniz Technical University and Gazi University respectively. He worked as research assistant at Bolu Abant Izzet Baysal University, between 2005-2006 and at Gazi University between 2006-2007. He has been working at Izmir Institute of Technology till 2007. His research interests are robotics, microprocessors and biomedical image analysis. 